\title{
Yield of MRI, high-density electric source imaging (HD-ESI), SPECT and PET in epilepsy surgery candidates
}

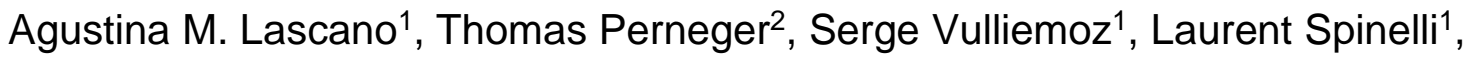 \\ Valentina Garibotto ${ }^{3}$, Christian M. Korff ${ }^{4}$, Maria I. Vargas ${ }^{5}$, Christoph M. Michel ${ }^{6}$, \\ Margitta Seeck ${ }^{1}$
}

${ }^{1}$ Department of Neurology, University Hospitals of Geneva, Switzerland

${ }^{2}$ Division of Clinical Epidemiology, University Hospitals of Geneva, Switzerland

${ }^{3}$ Department of Nuclear Medicine, University Hospitals of Geneva, Switzerland

${ }^{4}$ Pediatric Neurology, Child and Adolescent Department of Pediatrics, University Hospitals of Geneva, Switzerland

${ }^{5}$ Department of Neuroradiology, University Hospitals of Geneva, Switzerland

${ }^{6}$ Functional Brain Mapping Laboratory, Department of Neurology, University Hospitals of Geneva and University Medical Centre, Switzerland

\section{Corresponding author:}

Prof. Margitta Seeck

EEG and Epilepsy Unit, Department of Neurology, University Hospitals of Geneva, Rue Gabrielle-Perret-Gentil 4, 1211, Geneva, Switzerland

Tel.: +4122 3728476

Fax: +41223728476

E-mail: Margitta.Seeck@hcuge.ch 


\section{Abstract}

Objective: Preoperative workup aims at localizing the epileptogenic focus to achieve postoperative seizure-freedom. We studied the predictive value of non-invasive techniques, i.e. structural magnetic resonance imaging [MRI], high-density electric source imaging [HD-ESI] and metabolic imaging (positron emission tomography [PET]; single-photon emission computed tomography [SPECT]), in surgically treated patients.

Methods: A prospective study of 190 epileptic operated patients, with $>12$ months follow-up and analyzed with state-of-the-art algorithms. 58 patients underwent all techniques. We computed sensitivity, specificity, predictive value and diagnostic odds ratio $(\mathrm{OR})$ in relation to postoperative outcome.

Results: Of 190 patients, 148 (77.9\%) were seizure-free at follow-up. Resection of the epileptogenic focus was associated with favorable postsurgical outcome $(p<0.05)$. Among 58 patients who underwent all tests, only MRI and HD-ESI were favorable outcome predictors (MRI: OR 10.9, $\mathrm{p}=0.004$; HD-ESI: OR 13.1, $\mathrm{p}=0.004$ ). Patients with concordant structural MRI and HD-ESI results had 92.3\% (24/26) probability of favorable outcome. When both results were negative, probability was $0 \%(0 / 5)$; and when they disagreed, it was $63.0 \%(17 / 27)$.

Conclusions: Combination of MRI and HD-ESI offered the highest predictive value for postoperative seizure-freedom.

Significance: This finding highlights the added value of HD-ESI in the presurgical workup, in particular in combination with an informative MRI. 
Keywords: Presurgical evaluation; temporal lobe epilepsy; drug-resistant epilepsy; seizure-freedom; non-invasive procedures.

\section{Abbreviations}

$\mathrm{EEG}=$ Electroencephalography; SPECT = Single-photon emission-computed tomography; PET = Positron emission tomography; $\mathrm{MRI}=$ Magnetic resonance imaging; $\mathrm{ESI}=$ Electric source imaging. 


\section{Introduction}

Evaluation and management of drug-resistant seizures remains a challenging clinical problem in epilepsy. Notwithstanding the advent of new antiepileptic drugs, nearly one-third of patients with recently diagnosed epilepsy will not achieve seizure remission with pharmacological therapy (Kwan and Sander, 2004). On the other hand, epilepsy surgery in suitable candidates can eradicate or markedly reduce the frequency of seizures in about $\sim 60-70 \%$ of patients (Engel, 1993; Wiebe et al, 2002; Kwan and Sperling, 2009) and, therefore, should be considered early in the disease. Epilepsy surgery requires precise estimation of the epileptogenic zone to be removed in order to obtain seizure-freedom, while preserving the eloquent cortex (Rosenow and Luders, 2005). This is achieved by adding different non-invasive exams (e.g.: video-electroencephalogram $[E E G]$, magnetic resonance imaging [MRI], positron emission tomography [PET] and single-photon emission computed tomography [SPECT]) (Knowlton et al, 2008; Schramm and Clusmann, 2008). In some patients, intracranial EEG monitoring is necessary (Rosenow and Luders, 2001; Knowlton et al., 2008; Schramm and Clusmann, 2008; Yuan et al., 2012).

Few studies including large cohorts (i.e. > 50-60 patients) have compared the contribution of non-invasive neurophysiologic techniques in the presurgical work-up. A study of 62 patients undergoing intracranial EEG identified a positive predictive value of $78 \%$ for preoperative magnetic source imaging based on magnetoencephalography (Knowlton et al., 2006), with a sensitivity of 55\%. A recent prospective study compared electric source imaging (ESI) in a group of 152 operated patients with a follow-up period of $>1$ year and found highest sensitivity and specificity (> 80\%) for ESI based on EEG with high-density electrode recordings (HDESI) (Brodbeck et al., 2011). 
The aim of the present study, which is an extension of the prospective and methodological study performed by Brodbeck et al. (2011), was to determine which procedure or combination of procedures is the most predictive of seizure-free outcome in patients undergoing epilepsy surgery. Secondly, we consider only HDESI, given that its superiority over ESI with low electrode counts has been already shown (Brodbeck et al., 2011).

\section{Methods}

\section{Patient population}

This study was based on a prospectively collected database, including 190 patients who underwent surgery for medically intractable epilepsy at the University Hospitals of Geneva, Switzerland, between 1995 and 2012. All patients had a postsurgical follow-up period of at least 12 months. This study was approved by the local Ethics Committee, in agreement with the Declaration of Helsinki.

Presurgical workup included a thorough neurological examination, a neuropsychological assessment, a psychiatric evaluation, a high-resolution MRI, and in most cases PET. Ictal SPECT and ESI was added whenever possible. Forty-three patients underwent additional intracranial EEG recordings prior to surgical resection, if seizure onset zone could not be determined by non-invasive methods and/or to localize eloquent cortex. Out of those patients who underwent all non-invasive imaging exams ( $\mathrm{N}=58), 23$ were implanted with intracranial electrodes.

\section{Surgery}

Surgical decision-making was carried out on an individual basis at our weekly epilepsy surgery consensus conference. Resection was based on concordant 
information provided by EEG, neuroimaging techniques, observation of ictal semiology and neuropsychological tests. No rules were applied in prioritizing a certain modality. Whenever possible, surgery was performed with the aid of imaging guidance.

Surgical treatment included the resection of the temporal lobe $(n=125 ; 66 \%)$, the extra-temporal structures $(n=60 ; 31.5 \%)$ and, in a single case, a cerebellar lesion $(n=1 ; 0.5 \%)$. Transventricular hemispherotomy was applied in four cases $(2 \%)$. Out of the 125 patients who underwent temporal lobe surgery, $n=21$ were limited to the medial temporal lobe only, $\mathrm{n}=20$ to the lateral temporal lobe only, and $\mathrm{n}=84$ included both regions. Extra-temporal lobe surgery comprised unilobar $(n=31$; out of which $52 \%$ targeted the frontal lobe) or multilobar $(n=29 ; 48 \%)$ resections. MRI was abnormal in 174 patients.

\section{Study variables}

The outcome variable was the postoperative seizure outcome as determined by the neurosurgeon, neurologist or neuropediatrician on the last follow-up, according to Engel's classification (Engel et al., 1993). Table 1 provides information on the operated site and the postoperative outcome in 190 patients. Predictor variables were the results of preoperative imaging procedures described below.

\section{High Density Electric Source Imaging (HD-ESI)}

ESI based on EEG with a high number of electrodes was obtained in 85 patients by recording for 2-24 hours with 64 ( $n=2$ infants), 128 ( $n=44)$ or 256 electrodes ( $n=39)$ using HydroCel Geodesic Sensor Net (Electrical Geodesics Inc., Eugene, OR, USA). EEG was continuously recorded (1-2 h sessions) at a sampling rate of $1 \mathrm{kHz}$ and 
band-pass filtered between $0.1-400 \mathrm{~Hz}$. The Vertex $(\mathrm{Cz})$ electrode was used as recording reference and the data were referenced offline to the average reference. EEG was analyzed by means of a semi-automatic procedure composed by several steps described in Brodbeck et al. (2011).

The most dominant and isolated IEDs (i.e. spikes or sharp waves), with similar localisation and morphology, were visually detected by a blinded board certified EEG reader [A.M.L.]. Results were compared to those obtained by two unblinded authors in charge of the patients [M.S. and S.V.]. Numbers of averaged spikes in high-density EEG were on average 27 spikes (SD 34.26).

Interictal epileptic discharges (IED) were averaged across epochs of 500ms using the free academic software Cartool (D. Brunet, Geneva University Medical Center, Center for Biomedical Imaging, Geneva, Switzerland; http://sites.google.com/site/fbmlab/cartool). A band-pass filter of $0.1-30 \mathrm{~Hz}$ was applied to the on-going EEG. The EEG map at the $50 \%$ rising phase of the averaged IED was selected for further source localization.

A local autoregressive average (LAURA) distributed linear inverse solution (Grave de Peralta Menendez et al., 2001; Michel et al., 2004) was applied to estimate the intracranial 3D current density distribution of the averaged IED. We applied a simplified realistic head model based on the individual MRI which constraints the solution space to the grey matter (for methodological details see (Michel et al., 2004; Spinelli et al., 2000).

\section{Magnetic Resonance Imaging}

MRI was acquired either with a 1.5T Achieva (Phillips Healthcare, Netherlands) or a 3T Trio scanner (Siemens AG, Germany) and performed according to a standardized 
state-of-the-art epilepsy protocol (Vargas et al., 2013) using a 32-channel brain coil: a) coronal $\mathrm{T}_{2}$-weighted fast spin-echo repetition time (TR) $7520 \mathrm{~ms}$; echo time (TE) $114 \mathrm{~ms}$; voxel size $0.5 \times 0.4 \times 3 \mathrm{~mm}$ (slice thickness); b) sagittal 3D FLAIR (fluidattenuated inversion recovery) TR5000; TE419; inversion time (TI) 1800;isotropic voxel size $0.9 \times 0.9 \times 0.9 \mathrm{~mm}$ ); c) sagittal 3D gradient echo T1TR1750ms; TE2.29ms; isotropic voxel size $0.7 \times 0.7 \times 0.7 \mathrm{~mm}$; d) diffusion-weighted imaging (DWI) TR 8000ms; TE 84ms; 30 directions; e) and arterial spin labelling (ASL) TR 4000ms; TE $12 \mathrm{~ms}$; voxel size $3.4 \times 3.4 \times 4 \mathrm{~mm}$ (slice thickness). MRls were described by a boardcertified neuroradiologist with long experience in epilepsy imaging [MIV]. In those patients who had no visible lesion on the MRI, voxel-based comparison with a healthy control group was carried out, as described elsewhere (Huppertz et al., 2009).

\section{Positron Emission Tomography}

PET acquisition was performed on a BiographHiRez Sensation 16 (Siemens Healthcare, Erlangen, Germany) using a standard protocol recommended by the manufacturer. PET scans were carried out in 185 patients, during the interictal state, using $\left[{ }^{18} \mathrm{~F}\right]$-labeled fluorodeoxyglucose (FDG). The administered activity was 250 MBq for adults and was adapted to body weight for children. Images were acquired approximately 30 min after tracer administration.

FDG-PET images of a subgroup of adult patients ( 58 subjects who underwent all four imaging techniques) were also analyzed by voxel-wise comparison with a normal database using BRASS ${ }^{\text {TM }}$ automated functional brain analysis software (Hermes BRASS software, Nuclear Diagnostics AB, Sweden) (Slomka et al., 2001). Each 
individual FDG-PET image was warped to the reference template and a threshold of 2 SD was set to identify deviations from normal distribution (Radau et al., 2001).

Areas with minimal-to-low grade FDG uptake were visually identified by a boardcertified nuclear medicine specialist [V.G.], who interpreted also the SPECT studies (see below).

\section{Ictal Single-photon Emission-Computed Tomography}

Ictal SPECT scans was performed using a single bolus injection of $740 \mathrm{MBq}(20 \mathrm{mCi})$ ethlenecysteinate dimer labeled with $[99 \mathrm{mTc}]$ during seizure. The administered activity was $740 \mathrm{MBq}$ for adults and adapted to body weight for children. Scans were acquired 20-60 min after the radioisotope injection using a three-head gamma camera (Toshiba CGA-9300, Tokyo, Japan). A total of 137 patients were scanned. Focus localization was determined by visual analysis of the ictal exam. Ictal/Interictal SPECT were analyzed by subtraction ictal SPECT coregistered to MRI (SISCOM).

\section{Test Scoring}

After data acquisition and processing of all four modalities (MRI $n=190$, PET $n=185$, ictal SPECT $n=163, H D-E S I n=82$ ), localization findings were defined at a sublobar level. A visual selection method was equally applied across all four techniques. A score was established to assess the degree of localization of the probable seizureonset zone (Luders et al., 2006): 1) Localized within the resected lobe; 2) Localized both within and outside boundaries of the operated lobe; 3) Localized outside the resected lobe; 4) Normal/No pathology detected; 5) Exam not performed. For statistical analysis, patients from group 2), 3) and 4) were considered together and 
compared to group 1 (Table 2). Consensus was reached by a reviewer who was blinded to the final diagnosis [A.M.L.].

\section{Statistical analysis}

We defined a test as being positive if it placed the epileptogenic focus within the resection zone (i.e.: with no activity outside this area). For each test, we computed the proportion of seizure-free patients (Engel Class I) among those with a positive and a negative result, and obtained sensitivity (Sn), specificity (Sp), positive predictive value (PPV), negative predictive value (NPV), and prognostic odds ratio (OR) with its p-value. The prognostic OR can be defined as either $\frac{S n \cdot S p}{(1-S n) \cdot(1-S p)}$ or $\frac{P P V \cdot N P V}{(1-P P V) \cdot(1-N P V)}$; it provides a summary assessment of the predictive ability of a test. We repeated test comparisons in a subset of 58 patients who had available results for the four imaging methods (HD-ESI, MRI, PET and ictal SPECT). Table 3 details the clinical characteristics on that group of patients.

Regarding EEG recordings in the group of 58 patients: $n=2$ were recorded with 64 electrodes, $n=31$ using 128 channels and $n=25$ with 256 electrodes.

We also examined the performance of different tests combination; where having two positive results was compared to any other combination (e.g., comparison of both positive HD-ESI and MRI versus $1 / 2$ or both tests negative). Finally, we used multiple logistic regressions to identify test results that best predicted a seizure-free outcome. The analyses were conducted using IBM SPSS software version 18.

\section{Results}




\section{Patients}

Of 190 patients, 91 (47.9\%) were women. The median age at epilepsy onset was 7.0 +/- 10.8 years (range $0-54$ ), and age at surgery was $25.3+/-15.3$ years (range 0.660). Fifty-seven (30.0\%) patients were $<16$ years at time of surgery (median age: 8.6 $+/-4.6$ years).

\section{Post-surgical outcome}

At 12-moths follow-up, seizure outcome was Engel Class I in 148 (77.9\%) out of the 190 patients, Engel Class II in 17 (8.9\%), Engel Class III in 19 (10.0\%) and Engel Class IV in 6(3.2\%) (Table 1). Mean follow-up duration was 26.6 +/- 27 months. In our sample, patients suffering from extra-temporal lobe epilepsy presented a less favorable postoperative outcome (14/60 were Class III and 3/60 were Class IV), than patients with a temporal lobe intervention $(p=0.0002)$.

\section{Performance of individual tests}

All 190 patients had preoperative EEG recordings and standard MRI data. The number of patients with unavailable results was five for PET scan, 53 for ictal SPECT and 108 for HD-ESI (Table 2). If we detail the test scoring outcome per technique: a) MRI presented 132/190 results with score 1, 38/190 with score 2 and 20/190 with score 3+4; b) PET had 120/185 results with score 1, 45/185 with score 2 and 20/185 with score $3+4$; c) Ictal SPECT presented 75/137 with score 1, 41/137 with score 2 and 21/137 with score $3+4$; d) HR-ESI had 68/82 with score 1and 14/82 with score 3 . In the whole sample, all tests performed were associated $(p<0.05)$ with complete seizure control, defined as Engel Class I. The contrast was strongest for MRI, PET 
and HD-ESI $(p<0.01)$. No imaging technique had both high sensitivity and high specificity.

We then compared test performance indicators among the group of 58 patients who had all tests available (Table 4, upper half). Among these patients, 35 (67.3\%) were seizure-free. Only MRI and HD-ESI had prognostic OR greater than 5, and both were statistically significant even in this subsample ( $p=0.016$ and $p=0.011$, respectively).

\section{Performance of test combinations}

As expected, the combination of two positive tests identified fewer seizure-free patients than each positive test alone. Therefore, this resulted in lower values of sensitivity and negative predictive value, and higher values of specificity and positive predictive value (Table 4, lower half). The highest prognostic OR was obtained for the combination of positive results on the HD-ESI and the MRI.

Logistic regression modeling among the 58 patients with all tests confirmed that only $\mathrm{MRI}$ and HD-ESI were independent predictors of favorable outcome. Once these two predictors were included in the model, none of the other tests were statistically significant. The adjusted prognostic OR was 13.1 for HD-ESI and 10.9 for MRI (both $\mathrm{p}=0.004)$.

The combination of MRI and HD-ESI provided clinically useful results in more than half of the patients (Table 5). In a minority of patients $(5 / 58)$, both tests were negative, and none of them experienced a favorable clinical outcome (i.e. NPV of $100 \%)$. In about half of the patients (26/58), both tests were positive, and $92.3 \%$ (24/26) were seizure-free at follow-up. If only one of them was positive and the other negative (27/58), the proportion of seizure-free was $63.0 \%(17 / 27)$. 
We attempted to identify additional predictors of excellent outcome among the 27 patients with contradictory results on the MRI and HD-ESI. None of the nuclear imaging modalities (PET, SPECT) were associated with a favorable postoperative outcome ( $p>0.4)$.

\section{Discussion}

In this study, all preoperative imaging exams were associated with favorable postoperative outcome, but the strongest independent indicators were MRI and HDESI (independently or in combination; Figure 1).

MRI is capable of providing very high spatial resolution, and it is well known that the presence of a lesion is related to better outcome compared to non-lesional epilepsy. The definition of "non-lesional" varies across studies, and may refer either to imaging or histopathological results (Tellez-Zenteno et al., 2010), or may even include patients with hippocampal sclerosis (Lowe et al., 2010). In the present study, we adopted the very conservative and clinically practical definition of "non-lesional"; referring to the absence of a lesion in a high-resolution MRI obtained with an established "epilepsy protocol" including negative findings in a voxel-based comparison with a data set of $>300$ healthy subjects (Huppertz et al., 2005). Using this definition, MRI rendered a $70 \%$ sensitivity and specificity in the subset of 58 patients in whom all tests were performed. This may appear low, but in our population, many patients had multifocal, bilateral or no MRI lesion. Furthermore, even when MRI depicts a unifocal abnormality, the true epileptogenic zone may be more complex. A recent study on 158 patients with frontal lobe epilepsy, of which $76 \%$ were lesional cases, only $44 \%$ were postoperatively seizure-free (Simasathien et al., 2013). While incomplete resection is foreseeable when the lesion/focus is too 
close to eloquent cortex; in many cases, like in dysplastic lesions, the epileptogenic focus may not necessarily overlap with the visible structural anomaly. Interestingly, in all 158 patients, intracranial exploration was carried out indicating once more that the use of intracranial EEG is no guarantee of success.

Concerning the remaining techniques, sensitivity values for PET of $60-100 \%$ and ictal SPECT (with or without statistical analysis) of 66-97\% were reported (Knowlton, 2006; la Fougere et al., 2009). In the present study we obtained different results with ictal SPECT portraying the lowest sensitivity among all tests $(48.6 \%)$, followed by PET (62.9\%), maybe due to a higher percentage of patients with extratemporal foci as opposed to other studies. In MRI-negative temporal lobe epilepsy, a clear unilateral anterior temporal hypometabolism on PET has a similar high rate of postoperative seizure-freedom than hippocampal sclerosis (Carne et al., 2004).

HD-ESI represents focus localization in the 3-dimensional space within the individual patient's MRI. The combination of HD-ESI and MRI achieved a remarkable predictive capacity (i.e. seizure-free) in more than $90 \%$ of the patients $(24 / 26,92.3 \%)$ for whom results were concordant; but remained inconclusive for the remainder (17/32, 53\%). When neither test was able to locate the epileptogenic zone within the resected area, the results were uniformly unfavorable $(100 \% \mathrm{NPV})$.

Spatial precision of HD-ESI is in the cm-range. It has been shown that localization precision, to a sub-lobar level, can be obtained by using an electrode setup of more than 63 channels. Further increase from 64 to 128 electrodes showed very little improvement (Lantz et al., 2003). In addition, according to an earlier study from our group on 38 patients undergoing intracranial EEG monitoring, the median distance from the ESI maximum to the nearest electrode involved in the seizure onset was 15 $\mathrm{mm}$, which in most patients co-localized with the intracranial ictal focus (Megevand et 
al., 2014). This is relatively high, given that intracranial EEG alone is probably an imperfect "gold standard" due to potential spatial undersampling and an interelectrode distance of already $10 \mathrm{~mm}$. These results as well of studies with simultaneous intra- and extracranial EEG recordings also suggest that ESI is capable to detect deeper sources (Kossler et al., 2015; Ramantani et al., 2014).

In patients with discrepant MRI and HD-ESI results, nuclear imaging techniques did not add additional outcome predictors. Further research should focus on this group of patients, perhaps by including HR ictal recordings or research on other epilepsy markers like high frequency oscillations (Jacobs et al., 2010) or imaging changes of ictal rhythmic discharges (Yang et al., 2011). As opposed to interictal discharges, ictal activity is supposed to correspond with the underlying seizure onset area, although our and others' studies (Noe et al., 2013) point to an important role of correct and precise localization of interictal discharges. Systems allowing overnight long-term recordings with 128 or 256 channels maybe able to add HR-ictal recordings. Ictal or interictal, electromagnetic imaging offers a unique non-invasive opportunity to visualize neuronal discharges with both a high temporal and spatial resolution and thus, seems to be the most adequate technique in localizing the epileptogenic focus.

\section{Conclusions}

Our findings indicate that the use of MRI and ESI in the presurgical workup is associated with optimal seizure control, provided that both techniques concord and that the resection procedure takes the results into consideration. ESI together with MRI, predict the postoperative outcome. 


\section{Conflict of Interest}

M.S. received speaker's fees from UCB, EISAI, EGI and GSK; S.V. from UCB and EGI. C.K. received research fees from UCB. All authors have directly participated in the planning, execution or analysis of the manuscript.

\section{Acknowledgements}

This work was supported by the Swiss National Science Foundation [grants $\mathrm{n}^{\circ} 320030-146633$ to K.S., $\mathrm{n}^{\circ} 33 \mathrm{CM} 30-140332$ to M.S. and C.K. and $\mathrm{n}^{\circ} 141165$ to

S.V.]. Cartool software is developed by Denis Brunet, from the Functional Brain Mapping Laboratory, Geneva, supported by the Center for Biomedical Imaging (CIBM), Geneva and Lausanne, Switzerland. We would like to thank Dr. Verena Brodbeck, Dr Shahan Momjian and Prof. Karl Schaller for their profound contribution in this research study. 


\section{References}

Brodbeck V, Spinelli L, Lascano AM, Wissmeier M, Vargas MI, Vulliemoz S, et al. Electroencephalographic source imaging: a prospective study of 152 operated epileptic patients. Brain 2011;134:2887-2897.

Carne RP, O'Brien TJ, Kilpatrick CJ, MacGregor LR, Hicks RJ Murphy MA, et al. MRInegative PET-positive temporal lobe epilepsy: a distinct surgically remediable syndrome. Brain 2004;127:2276-2285.

Engel J Jr. Clinical neurophysiology, neuroimaging, and the surgical treatment of epilepsy. Curr Opin Neurol Neurosurg 1993;6:240-249.

Engel J Jr, Van Ness P, Rasmussen TB, Ojemann LM. Outcome with respect to epileptic seizures. In: Engel J Jr (ed). Surgical Treatment of the Epilepsies. New York: Raven Press 1993:609-621.

Grave de Peralta Menendez R, Gonzalez Andino S, Lantz G, Michel CM, Landis T. Noninvasive localization of electromagnetic epileptic activity. I. Method descriptions and simulations. Brain Topogr 2001;14:131-137.

Huppertz HJ, Grimm C, Fauser S, Kassubek J, Mader I, Hochmuth A, et al. Enhanced visualization of blurred gray-white matter junctions in focal cortical dysplasia by voxelbased 3D MRI analysis. Epilepsy Res 2005;67:35-50.

Huppertz HJ, Kurthen M, Kassubek J. Voxel-based 3D MRI analysis for the detection of epileptogenic lesions at single subject level. Epilepsia 2009;50:155-156.

Jacobs J, Zijlmans M, Zelmann R, Chatillon CE, Hall J, Olivier A, et al. High-frequency electroencephalographic oscillations correlate with outcome of epilepsy surgery. Ann Neurol 2010;67:209-220.

Knowlton RC, Elgavish RA, Bartolucci A, Ojha B, Limdi N, Blount J, et al. Functional imaging: II. Prediction of epilepsy surgery outcome. Ann Neurol 2008;64:35-41.

Knowlton RC. The role of FDG-PET, ictal SPECT, and MEG in the epilepsy surgery evaluation. Epilepsy Behav 2006;8:91-101.

Koessler L, Cecchin T, Colnat-Coulbois S, Vignal JP, Jonas J, Vespignani H, et al. Catching the invisible: Mesial temporal source contribution to simultaneous EEG and sEEG recordings. Brain Topogr 2015; 28: 5-20.

Kwan P, Sander JW. The natural history of epilepsy: an epidemiological view. J Neurol Neurosurg Psychiatry 2004;75:1376-1381.

Kwan P, Sperling MR. Refractory seizures: try additional antiepileptic drugs (after two have failed) or go directly to early surgery evaluation? Epilepsia 2009;50:57-62.

la Fougere C, Rominger A, Forster S, Geisler J, Bartenstein P. PET and SPECT in epilepsy: a critical review. Epilepsy Behav 2009;15:50-55.

Lantz G, Grave de Peralta R, Spinelli L, Seeck M, Michel CM. Epileptic source localization with high density EEG: how many electrodes are needed? Clin Neurophysiol 2003;114:63-69.

Lowe NM, Eldridge P, Varma T, Wieshmann UC. The duration of temporal lobe epilepsy and seizure outcome after epilepsy surgery. Seizure 2010;19:261-263.

Luders HO, Najm I, Nair D, Widdess-Walsh P, Bingman W. The epileptogenic zone: general principles. Epileptic Disord 2006;8:1-9.

Megevand P, Spinelli L, Genetti M, Brodbeck V, Momjian S, Schaller K, et al. Electric source imaging of interictal activity accurately localises the seizure onset zone. $J$ Neurol Neurosurg Psychiatry 2014;85:38-43.

Michel CM, Murray MM, Lantz G, Gonzalez S, Spinelli L, Grave de Peralta R. EEG source imaging. Clin Neurophysiol 2004;115:2195-2222. 
Noe K, Sulc V, Wong-Kisiel L, Wirrell E, Van Gompel JJ, Wetjen N, et al. Long-term outcomes after nonlesional extratemporal lobe epilepsy surgery. JAMA Neurol. 2013;70:1003-1008.

Radau PE, Slomka PJ, Julin P, Svensson L, Wahlund LO. Evaluation of linear registration algorithms for brain SPECT and the errors due to hypoperfusion lesions. Med Phys 2001;28:1660-1668.

Ramantani G, Dümpelmann M, Koessler L, Brandt A, Cosandier-Rimélé D, Zentner J, et al. Simultaneous subdural and scalp EEG correlates of frontal lobe epileptic sources. Epilepsia 2014; 55: 278-288.

Rosenow F, Luders H. Presurgical evaluation of epilepsy. Brain 2001;124:1683-1700.

Schramm J, Clusmann H. The surgery of epilepsy. Neurosurgery 2008;62:463-481.

Simasathien T, Vadera S, Najm I, Gupta A, Bingaman W, Jehi L. Improved outcomes with earlier surgery for intractable frontal lobe epilepsy. Ann Neurol 2013;73:646-654.

Slomka PJ, Radau P, Hurwitz GA, Dey D. Automated three-dimensional quantification of myocardial perfusion and brain SPECT. Comput Med Imaging Graph 2001;25:153164.

Spinelli L, Andino SG, Lantz G, Seeck M, Michel CM. Electromagnetic inverse solutions in anatomically constrained spherical head models. Brain Topogr 2000;13:115-125.

Tellez-Zenteno JF, Hernandez Ronquillo L, Moien-Afshari F, Wiebe S. Surgical outcomes in lesional and non-lesional epilepsy: a systematic review and meta-analysis. Epilepsy Res 2010;89:310-318.

Vargas MI, Becker M, Garibotto V, Heinzer S, Loubeyre P, Gariani J, et al. Approaches for the optimization of MR protocols in clinical hybrid PET/MRI studies. MAGMA 2013;26:57-69.

Wiebe S, Blume WT, Girvin JP, Eliasziw M. A randomized, controlled trial of surgery for temporal-lobe epilepsy. N Engl J Med 2001;345:311-318.

Yan L, Wilke C, Brinkmann B, Worrell GA, He B. Dynamic imaging of ictal oscillations using non-invasive high-resolution EEG. Neuroimage 2011; 56:1908-1917.

Yuan J, Chen Y, Hirsch E. Intracranial electrodes in the presurgical evaluation of epilepsy. Neurol Sci 2012;33:723-729. 


\section{Figure Legends}

Figure 1. Localization of non-lesional frontal epilepsy in a child. From left to right: magnetic resonance imaging (MRI), high-resolution electric source imaging (HD-ESI), positron-emission tomography (PET) and single-photon emission computed tomography (SPECT) localizing an anterior left frontal lesion. Dysplasia was confirmed by histopathological analysis. HD-ESI, PET and SPECT's results are projected on the individual anatomic brain. Sagittal (a) and longitudinal (b) slices are presented. This patient who had all concordant examinations was seizure free (Engel Class I) postoperatively after 3 years. 


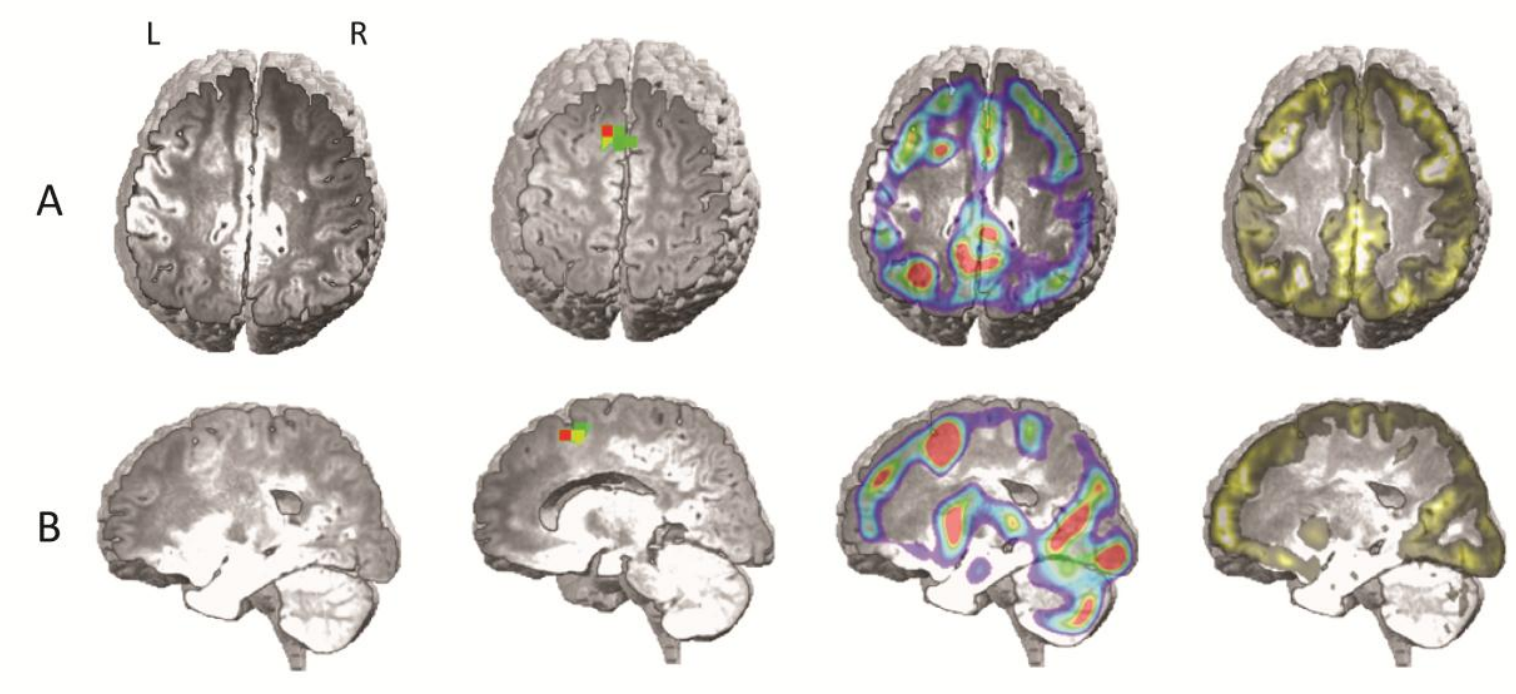


TABLE 1: Postoperative outcome of all 190 epileptic patients

\begin{tabular}{|c|c|c|c|c|c|}
\hline Operated Site & $\begin{array}{l}\text { Engel } \\
\text { Class I }\end{array}$ & $\begin{array}{l}\text { Engel } \\
\text { Class II }\end{array}$ & $\begin{array}{c}\text { Engel } \\
\text { Class III }\end{array}$ & $\begin{array}{c}\text { Engel } \\
\text { Class IV }\end{array}$ & TOTAL (\%) \\
\hline ETLE & 36 & 7 & 14 & 3 & 60 (31.5) \\
\hline \multicolumn{6}{|l|}{ TLE } \\
\hline MTLE & 16 & 4 & - & 1 & 21 (11) \\
\hline LTLE & 18 & 1 & 1 & - & $20(10.5)$ \\
\hline M/LTLE & 73 & 5 & 4 & 2 & $84(44.2)$ \\
\hline Subcortical & 1 & - & - & - & $1(0.5)$ \\
\hline Hemispherotomy & 4 & - & - & - & $4(2.1)$ \\
\hline ALL PATIENTS (\%) & 148 (77.9) & $17(8.9)$ & $19(10)$ & $6(3.2)$ & 190 \\
\hline
\end{tabular}

Engel Class I: seizure-free; II: decrease $>80 \%$; III: decrease $50-80 \%$; IV: decrease $<50 \%$; ETLE = Extratemporal lobe epilepsy; TLE = Temporal lobe epilepsy; MTLE = Mesiotemporal lobe epilepsy; LTLE = Latero-temporal lobe epilepsy. 
TABLE 2: Test results and seizure-free outcome in 190 patients

\begin{tabular}{|l|l|c|c|c|}
\hline & \multicolumn{1}{|c|}{ Result } & N (\%) & $\begin{array}{c}\text { Seizure } \\
\text { free, } \\
\text { N (row \%) }\end{array}$ & p-value \\
\hline MRI & Within resection & $132(69.5)$ & $116(87.9)$ & $<0.001$ \\
& Outside or normal & $58(30.5)$ & $32(55.2)$ & \\
\hline PET & Within resection & $120(63.2)$ & $102(85.0)$ & \multirow{2}{*}{0.006} \\
\hline HD-ESI & Outside or normal & $65(34.2)$ & $42(64.6)$ & \\
& Not done & $5(2.6)$ & $4(80.0)$ & $<0.001$ \\
& Within resection & $68(35.8)$ & $57(83.8)$ & \\
\hline
\end{tabular}

HD-ESI = Electric source imaging high-rdensity; MRI = Magnetic Resonance Imaging; SPECT = Single-photon Emission Computerized Tomography ; PET = Positron Emission Tomography. 
TABLE 3: Clinical characteristics of the subset of 58 patients

Sex ratio (M/F) 27/31

Mean age at disease onset $\quad 8.95$

Mean age at operation $\quad 24.76$

$\begin{array}{ll}\text { ETLE/TLE ratio } & 1: 1\end{array}$

Aetiology/Syndrome

Hippocampal Sclerosis $\quad 19$

Developmental§ 6

$\begin{array}{ll}\text { Tumor* } & 7\end{array}$

Lesional\& $\quad 7$

Gliosis $\quad 2$

Tuberous sclerosis $\quad 7$

Miscelaneous $^{\wedge}$

Non-lesional $\quad 8$

Mean post-op follow-up period (months) 22.6

Post-operative outcome (Engel Class)

I 40

II 9

III

IV 2

EEG

Number of electrodes

64 channels 2

128 channels 31

256 channels 25

Intracranial recordings $\quad 23$ 
§Focal cortical dysplasia, heterotopia, schizencephaly, lissencephaly, polimicrogyria *DNET, hamartoma, ganglioglioma, meningioma

\&Aneurysm, cyst, stroke

$\wedge$ Head trauma, post-infection 
TABLE 4: Test performance in 58 patients in whom all methods were performed, for single tests and for pairs of positive results

\begin{tabular}{|lccccc|}
\hline \multicolumn{1}{|c}{ Test } & Sensitivity (\%) & Specificity (\%) & PPV (\%) & NPV (\%) & OR \\
\hline MRI & 70.7 & 70.6 & 85.3 & 50 & 5.8 \\
PET & 65.9 & 58.8 & 79.4 & 41.7 & 2.8 \\
SPECT & 53.7 & 70.6 & 81.5 & 38.7 & 2.8 \\
HD-ESI & 87.8 & 47.1 & 80 & 61.5 & 6.4 \\
HD-ESI + MRI $^{*}$ & 58.5 & 88.2 & 92.3 & 46.9 & 10.6 \\
HD-ESI + PET* & 58.5 & 70.6 & 82.8 & 41.4 & 3.4 \\
HD-ESI + SPECT* & 43.9 & 82.4 & 85.7 & 37.8 & 3.7 \\
MRI + PET* $^{*}$ & 56.1 & 76.5 & 85.2 & 41.9 & 4.2 \\
MRI +SPECT* & 48.8 & 88.2 & 90.9 & 41.7 & 7.1 \\
PET + SPECT* $^{*}$ & 46.3 & 88.2 & 90.5 & 40.5 & 6.5 \\
\hline
\end{tabular}

HD-ESI = Electric source imaging high-rdensity; MRI = Magnetic Resonance Imaging; SPECT = Single-photon Emission Computerized Tomography $; \mathrm{PET}=$ Positron Emission Tomography. PPV = Positive predictive value; NPV = Negative predictive value; $\mathrm{OR}=$ Odds ratio.

${ }^{*}$ Both tests positive versus any other result 
TABLE 5: HD-ESI and MRI in patients with all tests

\begin{tabular}{|c|c|c|}
\hline \multirow[b]{2}{*}{ Test results } & \multicolumn{2}{|c|}{ Patients with all tests perfomed } \\
\hline & $\mathbf{N}(\%)$ & $\begin{array}{c}\text { Seizure free } \\
\text { (row \%) }\end{array}$ \\
\hline Both Negative & $5(8.6)$ & $0(0.0)$ \\
\hline Only MRI positive & $8(13.8)$ & $5(62.5)$ \\
\hline Only HD-ESI positive & $19(32.7)$ & $12(63.2)$ \\
\hline Both positive & $26(44.8)$ & $24(92.3)$ \\
\hline TOTAL & $58(100 \%)$ & $41(69.5)$ \\
\hline
\end{tabular}

HD-ESI = High density electric source imaging; $M R I=$ Magnetic Resonance Imaging 